\title{
Clinical Significance and Therapeutic Potential of the Programmed Death Ligand-1 (PD-L1) and PD-L2 Expression in Human Colorectal Cancer
}

Martin Gasser ${ }^{1 \#}$, Matthias Koenigshausen ${ }^{2 \#}$, Martin R Grimm ${ }^{3}$, Claudia Stein ${ }^{4}$, Tanja Grimmig ${ }^{5}$, Martin Wagner ${ }^{5}$, Melissa Yeung ${ }^{6}$, Roberta $^{2}$ Rehder $^{4}$, Ekaterina Nichiporuk Stumpf ${ }^{5}$, Roman Moench ${ }^{5}$, Carmen M Ribas ${ }^{4}$, Sudipta Tripathi ${ }^{6}$, Anil Chandraker ${ }^{6}$, Uwe Maeder ${ }^{7}$, Uwe Heemann ${ }^{8}$, Christoph-Thomas Germer ${ }^{1}$ and Ana Maria Waaga-Gasser ${ }^{5,6^{*}}$

${ }^{1}$ Department of Surgery I, University of Wuerzburg, Wuerzburg, Germany

${ }^{2}$ Department of Trauma Surgery, Ruhr University Bochum, Bochum, Germany

${ }^{3}$ Department of Oral and Maxillofacial Plastic Surgery, University of Tuebingen, Tuebingen, Germany

${ }^{4}$ Medical School, Evangelic Faculty of Paraná, Curitiba, PR, Brazil

${ }^{5}$ Department of Surgery I, Molecular Oncology and Immunology, University of Wuerzburg, Wuerzburg, Germany

${ }^{6}$ Renal Division and Transplantation Research Centre, Brigham and Women's Hospital, Harvard Medical School, Boston, USA

${ }^{7}$ Interdisziplinäres Tumorzentrum, University of Wuerzburg, Wuerzburg, Germany

${ }^{8}$ Department of Nephrology, University of Munich, Klinikum Rechts der Isar, Munich, Germany

"Equally contributed

\begin{abstract}
Purpose: The programmed death-1/programmed death ligand (PD-1/PD-L) pathway in T cell activation has been shown to play an important role in tumor evasion from host immunity. The predictive value of PD-L1 and PDL2 expression in colorectal cancer (CRC) remains still under discussion. We analyzed whether negative signaling of infiltrating PD-1-positive T cells through PD-L1 and PD-L2 within the tumor could promote further tumor progression through downregulation of anti-tumor immunity.
\end{abstract}

Methods: We investigated PD-L1 and PD-L2 expression in tumors from patients with CRC and analyzed its prognostic significance with respect to outcome analysis.

Results: T cell infiltration was observed in $90.5 \%$ of the tumors, with $58 \%$ of the patients demonstrating PD-1positive T cells in their tumors. Patients who developed PD-1-positive T cell infiltration showed increased PD-L1expression within their tumors than PD-1-T cell negative individuals. Presence of tumor infiltrating PD-1-positive T cells was more pronounced in advanced stage cancers than in early cancers. Ligand expression (PD-L1/PD-L2) in the tumors combined with dense PD-1-positive T cell infiltration was associated with poor prognosis. Multivariate analysis demonstrated that PD-L expression in the tumors was an independent prognostic factor in CRC.

Conclusion: The presented results from primary tumors and CRC patient outcome analysis suggest that negative signaling of infiltrating PD-1-positive T cells through PD-L1 expression within the tumor may promote further tumor progression through downregulation of anti-tumor immunity. Co-expression of PD-1 on CD4/Foxp3positive $T$ cells was found indicating $T$ regulatory cell-mediated mechanisms by which tumor cells can evade immune recognition and destruction. This study demonstrates the importance of strategies inhibiting negative PD-1/ PD-L1 signaling in CRC.

Keywords: PD-1/PD-L signaling pathway; Tumor evasion; Immunotherapy; Tumor-infiltrating T lymphocytes; Colorectal cancer

\section{Introduction}

Colorectal cancer is one of the leading causes of cancer-associated mortality worldwide. Despite major advances in diagnosis and treatment of the disease, overall mortality is still high and needs further efforts to reduce cancer related death $[1,2]$. Currently, Dukes' or UICC classification are the most commonly used predictors of prognosis for CRC patients; indeed $30 \%$ to $40 \%$ of UICC stage III and IV patients experience relapse and die of the disease [1,2] as they often develop distant metastases in the liver or lung even after curative surgery. Although combinational pre-operative chemotherapy or radio chemotherapy with surgery show a survival benefit long-term outcome, in many patients still remains unfavorable [3-5]. Thus, novel strategies need to be developed and established in order to improve patients' prognosis.

The role of the immune system in the progression of malignancies currently gains further interest. Modulation of the anti-tumor immune response by the tumor cells is a critical mechanism implicated in evasion of the tumor from immune recognition, thus, influencing tumor progression and metastasis. It appears that an immune privileged microenvironment is formed around tumors, that protects malignant cells from immunological destruction [6-9].

Programmed death-1 (PD-1) is a co-inhibitory receptor expressed by antigen-stimulated $\mathrm{T}$ cells and functions as a major negative regulator of $\mathrm{T}$ cell immunity, to control peripheral tissue tolerance and immune homeostasis [10-12]. Two ligands for PD-1, PD-L1 (B7-H1, also known as CD274) and PD-L2 (B7-DC, also known as CD273) have been identified [13-17]. Previous studies have demonstrated that PD-1/ PD-L1 signaling inhibits T cell growth and cytokine release [14]. PD-L1

*Corresponding author: Prof. Dr. Ana Maria Waaga-Gasser, Department of Surgery I, Molecular Oncology and Immunology, University of Wuerzburg Oberduerrbacher Str. 6, D-97080 Wuerzburg, Germany, Tel: 4993120131715 Fax: 4993120131729; E-mail: waaga_a@ukw.de, awaaga@partners.org

Received July 10, 2017; Accepted August 09, 2017; Published August 14, 2017

Citation: Gasser M, Koenigshausen M, Grimm MR, Stein C, Grimmig T, et al (2017) Clinical Significance and Therapeutic Potential of the Programmed Death Ligand-1 (PD-L1) and PD-L2 Expression in Human Colorectal Cancer. Cancer Sci Ther 9: 566-573. doi: 10.4172/1948-5956.1000475

Copyright: $\odot 2017$ Gasser M, et al. This is an open-access article distributed under the terms of the Creative Commons Attribution License, which permits unrestricted use, distribution, and reproduction in any medium, provided the original author and source are credited. 
expressed on tumor cells increases apoptosis of antigen-specific T cell clones in vitro [18]. Furthermore, PD-L1 blockade with an anti-PD-L1 monoclonal antibody enhanced anti-tumor immunity and inhibited tumor growth in vivo [19]. Therefore, PD-L1 has been suggested to play an important role in immune evasion from the host immune system via PD-1-mediated inhibitory signals that give tumors advantage by selectively inhibiting CD8-positive $\mathrm{T}$ cells $[13,20,21]$. In addition, PD-L1 blockade prevents activation and expansion of CD4-positive $\mathrm{T}$ cells with regulatory functions. These studies have been well shown [22] conclusive results using murine tumor models and anti-PD-L1 antibody studies have previously been started to evaluate its clinical relevance in solid tumors $[18,20,22,23]$. Although PD-L1 expression has been reported in human cancers of the lung, ovary, colon, and in melanomas, its effect on patient's prognosis for CRC and lung cancer is still controversial $[18,24]$. On the other hand, the function of PDL2 in tumors remains largely unknown and only a few reports have suggested that PD-L2 may also play a role in tumor immunity $[25,26]$. It was shown that PD-L2 expression on the tumor cells promotes CD8 $\mathrm{T}$ cell-mediated rejection at both the induction and effector phase of antitumor immunity [25]. However, there is little information of PDL2 expression in tumors and its relevance in the clinical setting. In this study, we investigated the expression of PD-L1 and PD-L2 in human colorectal cancer to define their prognostic relevance in the context of PD-1 expression profiles on T cells.

\section{Materials and Methods}

\section{Patients}

This study prospectively enrolled consecutive patients that were undergoing elective surgery for primary CRC $(n=670)$ at the University Hospital of Wuerzburg from July 2003 to June 2008. Of these, 116 patients with a mean age of $66.1 \pm 5.6$ years were included that underwent curative R0 resections. Patients with secondary carcinoma were excluded. All patients were followed-up regularly at 3 months, 6 months and 12-month intervals in accordance with the guidelines of the German tumor centers (completeness index of 0.96) [4]. The follow-up of all patients comprised 60 months. The study was approved by the regional Ethics committee. Patients were assigned to UICC stages as followed: stage I, $n=20$; stage II, $n=27$; stage III, $\mathrm{n}=40$, and stage IV, $\mathrm{n}=29$. Tumors were evaluated for location, stage, and differentiation grade. Data concerning age, gender, postoperative course with follow up for the analysis of tumor recurrence and tumor related survival were collected in our database (oral resection margin). Tumor tissue samples as well as normal colon tissues from the patients were collected with informed consent immediately after surgical resection, frozen instantly in liquid nitrogen, and stored at $-80^{\circ} \mathrm{C}$ until further analysis. For immunohistochemistry, a part of fresh tumor tissue was immediately embedded in optimum cutting temperature compound and $5 \mu \mathrm{m}$ frozen sections were taken with a cryostat (Leica, Solms, Germany). Serial sections from each specimen were stained with $\mathrm{H}$ and $\mathrm{E}$ for histological evaluation.

\section{Antibodies}

The PD-1 (Programmed death-1) monoclonal antibody (mAb) was purchased from MBL (Naka-ku, Nagoya, Japan). PD-L1 and PD-L2 mAb were from eBioscience (Kranenburg, Germany). T cell subpopulations were identified by positive staining for CD4+ (T helper) and CD8+ (T cytotoxic) cells (DAKO, Glostrup, Denmark). For analysis of regulatory T cells Foxp3 (forkhead transcription factor) (Abcam, Cambridge, UK) was used. Isotype control mAbs were obtained from Pharmingen (Heidelberg, Germany). The secondary antibodies were horseradish peroxidase (HRP)-conjugated rabbit anti-mouse IgG and alkaline phosphatase (AP)-conjugated rabbit anti-mouse IgG purchased from DAKO (Glostrup, Denmark). Secondary antibodies used for immunofluorescence were Cy5-conjugated AffiniPure Donkey anti-goat IgG and FITC (Fluorescein-5-isothiocyanate)-conjugated AffiniPure Donkey anti-mouse IgG (Jackson ImmunoResearch Laboratories Inc., Suffolk, England).

\section{Immunohistochemistry}

To analyze the expression of PD-1, PD-L1, PD-L2, CD4, CD8, and Foxp3 in tumor specimens, we performed staining of these factors. Serial cryostat sections were performed followed by incubation of the sections with primary $\mathrm{mAb}$ or control $\mathrm{mAb}$ and with a secondary HRPconjugated antibody. The sections were then developed by incubation with 3.3'-diaminobenzidine (DAB) substrate (Biogenex, San Ramon, CA). PD-1 was analyzed on infiltrating T cells using sequential HRP/ AP immunoenzymatic double staining. Antibodies bound during the first staining step were blocked using EnVision K1395 double-stain Block (DAKO, Glostrup, Denmark) according to the manufacturer's instructions. The sections were subsequently incubated with the second primary $\mathrm{mAb}$ followed by incubation with an AP-conjugated secondary antibody and development by incubation with K1395 Fast Red (DAKO, Glostrup, Denmark) followed by counterstaining with hemalaun. The intensity of each immunoenzymatic staining on tumor cells in six individual magnified fields $(\times 400)$ for each staining was scored using a four-scale system. Intense staining in more than $50 \%$ of cells was considered as +++; staining present in a smaller proportion of the cells ( $10 \%$ to $50 \%)$ as ++ ; faint or light brown/red membranous or cytoplasmic staining in a few tumor cells $(<10 \%)$ was scored as + ; and no staining was scored as 0 . Analysis was performed independently by two observers in a blinded fashion. The sequential immunofluorescent double labelling was done by incubation with a Cy5-conjugated AffiniPure Donkey anti-goat IgG against the host species of the first primary antibody. The following second primary antibody was incubated with a FITC-conjugated AffiniPure Donkey anti-mouse IgG. After incubation with normal mouse serum (Biomeda, Burlingame, CA) diluted in TBS a PE (Phycoerythrin) labelled CD4 antibody was added. The sections were counterstained with DAPI $\left(4^{\prime}, 6^{\prime}\right.$-diamidino2-phenylindol, dihydrochloride, Invitrogen, Eugene, Oregon, USA) and mounted with Fluoromont (SouthernBiotech, Birmingham, USA).

\section{Real Time Polymerase Chain Reaction (Real Time PCR) for PD-1, PD-L1, PD-L2, and tumor-infiltrating $T$ cell subpopulation genes in colorectal tumor specimens}

mRNA expression of PD-1, PD-L1, PD-L2, CD4, CD8, Foxp3 was analyzed in colorectal cancer specimens by real time PCR. The primer sequences used were purchased from Sigma Genosys, Woodlands, TX. PCR thermal cycling conditions were as follows: $95^{\circ} \mathrm{C}$ for $10 \mathrm{~min}$, followed by 40 cycles of $95^{\circ} \mathrm{C}$ for 15 secs and $60^{\circ} \mathrm{C}$ for 60 secs. All samples were assayed in duplicate and normalized during data analysis. The average threshold cycle $(\mathrm{Ct})$ value was calculated as the cycle number at which fluorescence of reporter reaches a fixed threshold. The difference $(\Delta \mathrm{Ct})$ between the average $\mathrm{Ct}$ values of the samples in the target wells and those of the housekeeping gene, GAPDH, was assessed, followed by the calculation of the difference between the average $\Delta \mathrm{Ct}$ values of the samples for each target and the $\Delta \mathrm{Ct}$ value of the control sample (normal colon tissue, $\mathrm{n}=85)$ for that target $(\Delta \Delta \mathrm{Ct})$. The relative quantification value, fold difference, is expressed as $2^{-\Delta \Delta \mathrm{Ct}}$. Results were normalized to colon normal tissue (1.0) and expressed as fold difference. 
Citation: Gasser M, Koenigshausen M, Grimm MR, Stein C, Grimmig T, et al. (2017) Clinical Significance and Therapeutic Potential of the Programmed Death Ligand-1 (PD-L1) and PD-L2 Expression in Human Colorectal Cancer. Cancer Sci Ther 9: 566-573. doi: 10.4172/1948-5956.1000475

\section{Statistical Analysis}

The chi-square test was used for univariate analysis between PD-L1 and PD-L2 expression and clinicopathological data. The Kaplan-Meier method was performed to calculate the cumulative survival rates and to plot survival curves, the log rank test was used to find statistical differences between the curves. Tumor related survival was calculated from the time of surgery to the last contact or death. Relapse free time was calculated from the date of surgery to the date of first tumor relapse. Multivariate analysis by Cox regression was then performed to determine the most important predictors of survival among all of the possible variables and the associations of T cell PD-1 expression with outcome. $P$ values $<0.05$ were considered to be statistically significant, values $<0.01$ highly significant. All statistical analysis was conducted using the SPSS 12.0 for Windows statistical software package. The Mann-Whitney-U-Test was performed to compare the PD-1, PD-L1, and PD-L2 immunostaining, gene expression, and the development of postoperative metastases/recurrence in the 116 patients.

\section{Results}

\section{PD-L1 and PD-L2 expression in the tumor}

Of the 116 evaluated cancer tissues, 49 (42.2\%) were positive for PD-L1 expression at the protein level while 54 (46.6\%) showed significant gene expression; comparably 48 (41.4\%) tumors were positive for PD-L2 protein expression with 53 of the tumors $(45.7 \%)$

a

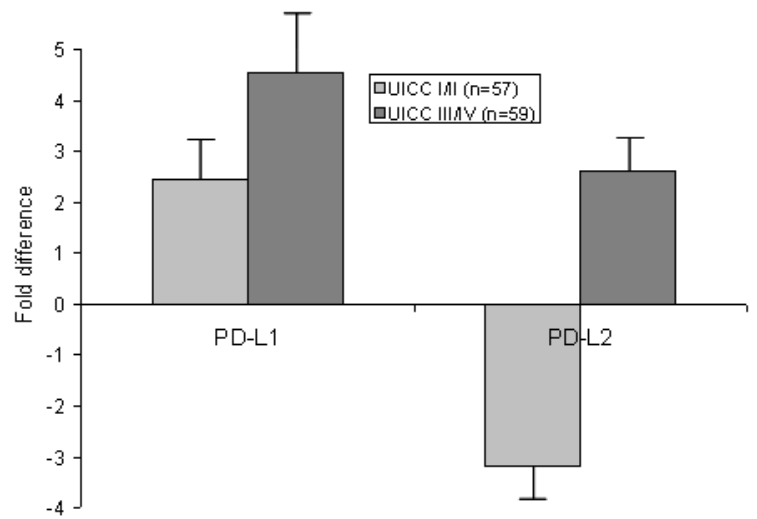

UICC II

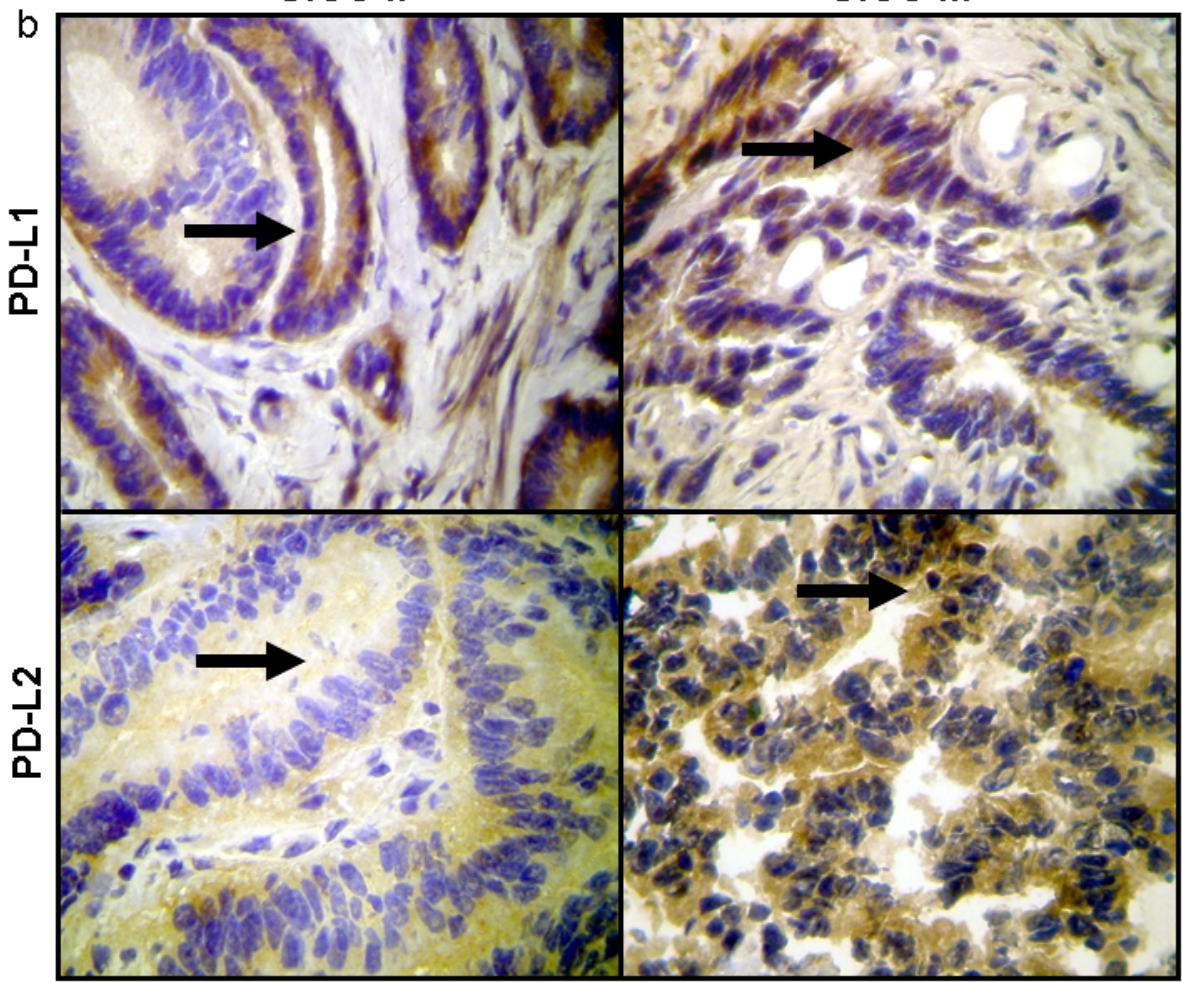

Figure 1: Quantification of PD-L gene expression by real-time quantitative PCR and immunohistochemical staining for human colorectal cancer. PD-L gene expression was significantly elevated at late stage of disease (UICC III/IV vs. I/II, $p<0.0001$ ). Relative quantification value, fold difference, is expressed as $2^{-\Delta \Delta C t}$ (a). Representative example of PD-L1 and PD-L2 protein expression at UICC stage II (top and bottom left, respectively) and at UICC stage III (top and bottom right, respectively) (b). PD-L1 and PD-L2 expression increased at the tumor site in patients of colorectal cancer at advanced stages as compared to early stages (UICC III/IV vs UICC I/II, p<0.0001). Original magnification $\times$ 400. Staining: 3.3'-diaminobenzidine (DAB), brown colour. 
demonstrating increased PD-L2 gene expression. In addition, 28 tumors $(24.1 \%)$ were positive for both PD-L1 and PD-L2 expression with significantly upregulated gene expression of the two ligands in advanced stage cancers (UICC III/IV vs. I/II, p<0.0001) (Figure 1a). Immunohistochemical analysis showed that both ligands, PD-L1 and PD-L2, were expressed mainly at the cell membrane but also stained positive in the cytoplasm of tumor cells (Figure 1b). Increased mRNA and protein expression in advanced but also in early stage cancers was positively correlated with each other for both PD-L1 $(\mathrm{p}=0.005)$ and
PD-L2 ( $\mathrm{p}=0.003$ ). For the following data analysis, we employed the quantified data of real-time PCR.

\section{Prognostic value of PD-L expression}

Overall survival of PD-L1- and PD-L2-overexpressing cancer patient's independent of their tumor stage (UICC I-IV) was significantly worse than that of negative patients $(\mathrm{p}=0.004$ and $\mathrm{p}=0.044$, respectively (Figures $2 \mathrm{a}$ and $2 \mathrm{~b}$ ). This was also observed for the second calculated parameter disease free survival $(\mathrm{p}=0.002$ and $\mathrm{p}=0.01)$. Moreover,

a

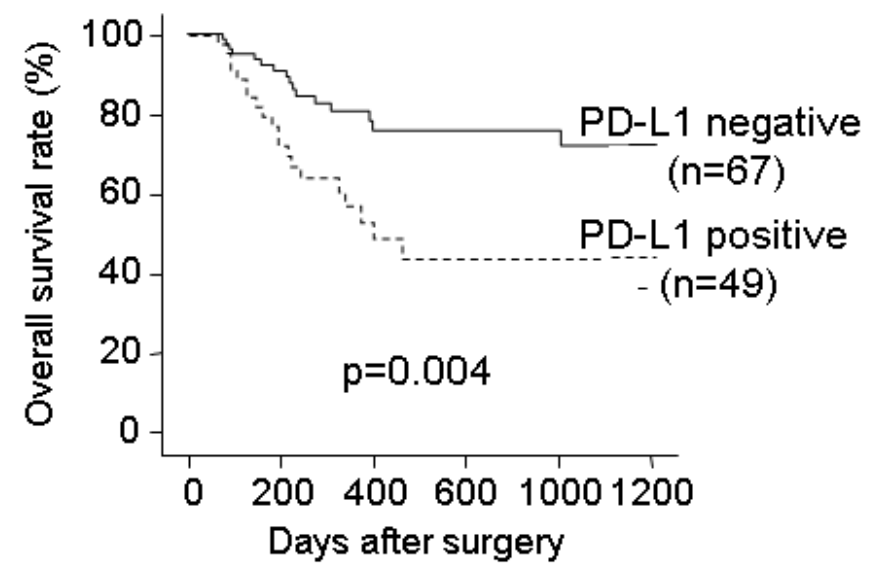

b

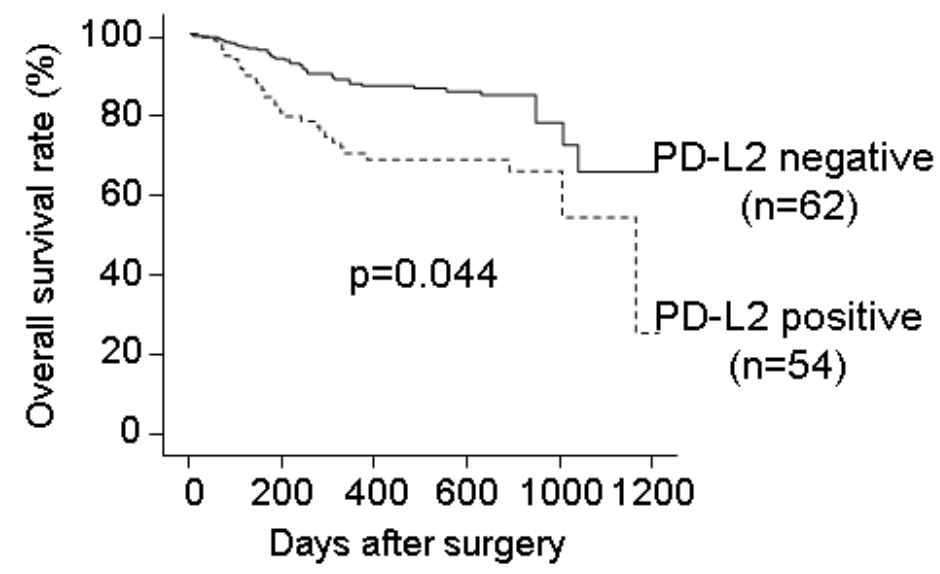

C

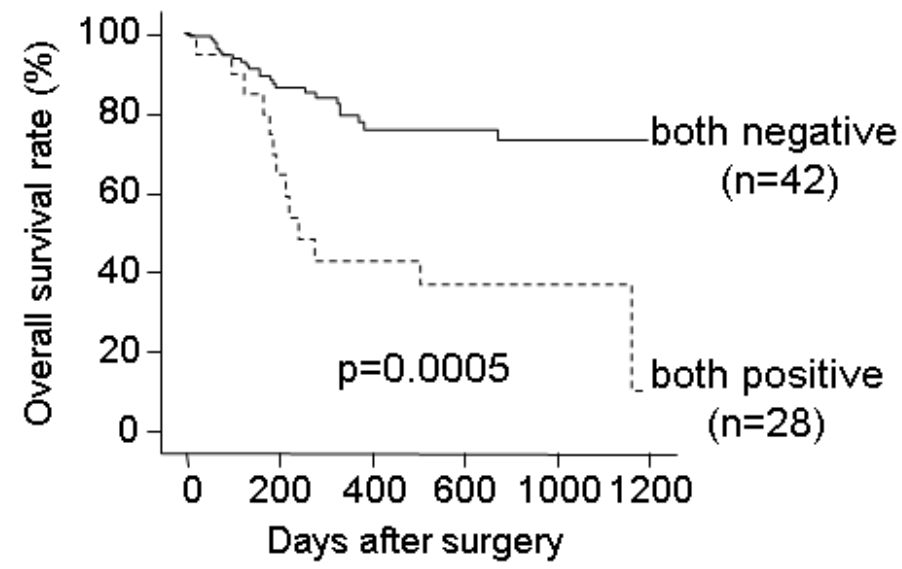

Figure 2: Overall survival of analyzed patients with colorectal cancer in relation to elevated PD-L gene expression within their tumors. PD-L1 and PD-L2-positive patients showed an inferior survival than negative patients at all stages (UICC stage I-IV, $p=0.004$ and 0.044). PD-L2-positive patients at UICC stage IV had an inferior survival than negative patients $(p=0.011)(a$ and $b)$. Patients positive for both PD-L1 and PD-L2 gene expression demonstrated a highly significant inferior survival than double negative patients (c). 
overall survival of patients with tumors overexpressing both genes PD-L1 and PD-L2 were even worse than that of patients with tumors not expressing significantly both ligands $(\mathrm{p}=0.0005)$ (Figure $2 \mathrm{c}$ ). The prognostic significance of PD-L gene expression was correlated with the protein expression profiles analyzed within the tumors. The overall survival of the 49 out the 116 patients $(42.2 \%)$ with cancers positive for PD-L1 protein expression was significantly worse than that of the remaining 67 patients with negative expression of the ligand in their tumors $(p=0.03)$. In addition, when compared with each other, PD$\mathrm{L} 1$ and $\mathrm{PD}-\mathrm{L} 2$, the 49 patients with tumors positive for PD-L1 protein expression had a worse overall survival than the 48 patients positive for PD-L2 $(\mathrm{p}=0.041)$.

Gene analysis showed significant worse overall survival for patients (completed follow-up of 60 months after surgery) with PD-L1 and PD-L2 overexpressing tumors when categorized by T category, N1/N2 category, M1 category as well as advanced UICC stages III/IV (Table 1). These results indicate that PD-L1 and PD-L2 expression was associated with a progressive course of the disease regarding nodal and metastatic status as well as UICC stages.

Cox regression analysis showed that PD-L overexpression in the tumor was an independent prognostic factor when considering overall survival $(\mathrm{p}=0.0002)$. Moreover, lymph node spreading (N1/2 status) was likewise an independent risk factor $(\mathrm{p}=0.001)$ while other factors were not significantly different.

\section{Correlation between PD-1 expression and tumor-infiltrating T lymphocytes}

We determined protein and gene expression levels of immune $\mathrm{T}$ cell-associated PD-1 expression and compared with the individual outcome of the patients. For this purpose, double immunostaining of PD-1 with markers for CD4+ and CD8+ immune cells were analyzed. Double positive $\mathrm{CD} 4+/ \mathrm{PD}-1+$ and/or $\mathrm{CD} 8+/ \mathrm{PD}-1+\mathrm{T}$ cells were found to be present in 67 (57.8\%) individual tumors. Among these cells the proportion of $\mathrm{PD}-1$ positive tumor infiltrating CD4+ T cells profoundly increased in late stage cancers (UICC stages III/IV versus I/II: $57.8 \%$ vs. $24.4 \%$, normal control $11.3 \%$, $\mathrm{p}=0.001$ (Figure $3 \mathrm{a}$ ). In contrast, the proportion of $\mathrm{PD}-1$ positive infiltrating CD8+ T cells decreased from early to late stages (UICC stages I/II vs. III/IV: $25.6 \%$ vs. $9.2 \%, \mathrm{p}=0.0005$ (Figure 3a).

To further analyze whether infiltration patterns of $\mathrm{T}$ cells with regulatory characteristics like the expression of Foxp3 was associated with the observed $\mathrm{CD} 4+/ \mathrm{CD} 8+\mathrm{T}$ cell infiltration profiles, coexpression studies of PD-1 with CD4+ and Foxp3 double positive $\mathrm{T}$ cells were performed using double and triple staining techniques. Expression of PD-1 on Foxp3+ CD4+ T cells was intensified in late stage cancers compared to early cancers and normal tissue (UICC stage III/IV vs. UICC I/II: $57.8 \%$ vs. $25.5 \%$, normal tissue $8.6 \%$ ) (Figure 3a) suggesting that the presence of such T cells with regulatory characteristics is associated with tumor progression. Real Time PCR analysis confirmed overexpression for PD-1, for CD4 as well as for Foxp3 gene overexpression in late stage cancers (UICC III and IV) as compared to early stage cancers (UICC I and II) $\mathrm{p}<0.005, \mathrm{p}<0.001$, and $\mathrm{p}<0.001$ respectively (Figure $3 \mathrm{~b}$ ). In contrast, CD8 gene expression was decreased in advanced stage cancers ( $p<0.0001$, UICC III and IV) (Figure 3). These latter results are in accordance with results obtained by immunohistochemical analysis and suggest that negative signaling via $\mathrm{PD}-1$ expressed on infiltrating immune cells (PD-1 positive CD8+ $\mathrm{T}$ cells) might be one mechanism of downregulation of the tumor immune response that is involved in the progression of the tumor.

Multivariate analysis showed that patients with tumors infiltrated by immune cells expressing PD-1 on their surface were significantly more likely to die from CRC as compared to patients without tumor infiltrating immune cells positive for PD-1 (risk ratio, 2.21; 93\% confidence interval, 1.21-3.72; $\mathrm{p}=0.003$ ). This indicates, that PD-1

\begin{tabular}{|c|c|c|c|c|c|c|c|}
\hline \multirow[t]{2}{*}{ Characteristics } & \multirow[t]{2}{*}{ Total (n) } & \multicolumn{3}{|c|}{ PD-L1 } & \multicolumn{3}{|c|}{ PD-L2 } \\
\hline & & Positive & Negative & $\mathbf{P}$ & Positive & Negative & $\mathbf{P}$ \\
\hline \multicolumn{8}{|l|}{ Gender } \\
\hline Male & 67 & 59.2 & 100 & 0.063 & 61.3 & 95.3 & \multirow[t]{2}{*}{0.091} \\
\hline Female & 49 & 78.2 & 80.3 & 0.143 & 58.6 & 98.9 & \\
\hline \multicolumn{8}{|l|}{ Age } \\
\hline$<65$ & 52 & 90.1 & 96.4 & 0.305 & 89.2 & 97.1 & \multirow[t]{2}{*}{0.283} \\
\hline$>65$ & 64 & 50.3 & 68.3 & 0.082 & 52.1 & 69.8 & \\
\hline \multicolumn{8}{|l|}{ Tumor status } \\
\hline $\begin{aligned} \mathrm{T} 1\end{aligned}$ & 28 & 100 & 100 & 0.801 & 100 & 100 & 0.801 \\
\hline T2 & 29 & 83.7 & 100 & 0.602 & 80.1 & 99.5 & 0.580 \\
\hline T3 & 29 & 52.4 & 91.3 & $\underline{0.024}$ & 48.1 & 99.5 & $\underline{0.021}$ \\
\hline T4 & 30 & 44.3 & 78.1 & $\underline{0.020}$ & 41.7 & 75.9 & $\underline{0.022}$ \\
\hline \multicolumn{8}{|l|}{ Nodal status } \\
\hline No & 57 & 100 & 89.3 & 0.484 & 81.4 & 100 & 0.201 \\
\hline N1 & 29 & 46.0 & 75.8 & 0.010 & 51.1 & 87.3 & 0.017 \\
\hline N2 & 30 & 39.2 & 61.1 & $\underline{0.013}$ & 40.6 & 64.9 & 0.019 \\
\hline \multicolumn{8}{|l|}{ Metastatic status } \\
\hline MO & 87 & 81.3 & 97.2 & 0.104 & 84.5 & 96.9 & 0.305 \\
\hline M1 & 29 & 14.3 & 29.5 & 0.009 & 21.4 & 34.6 & 0.041 \\
\hline \multicolumn{8}{|l|}{ UICC } \\
\hline 1 & 28 & 100 & 100 & 0783 & 100 & 100 & 0.808 \\
\hline II & 29 & 89.7 & 92.1 & 0.301 & 89.9 & 91.9 & 0.321 \\
\hline III & 30 & 41.2 & 66.3 & $\underline{0.024}$ & 53.7 & 68.7 & 0.058 \\
\hline IV & 29 & 18.9 & 37.5 & $\underline{0.014}$ & 26.2 & 38.4 & 0.049 \\
\hline
\end{tabular}

Table 1: Correlation between PD-L1 and PD-L2 expression status and 5-year-survival rate in combination with clinicopathologic characteristics in patients with colorectal cancer (in \%). 


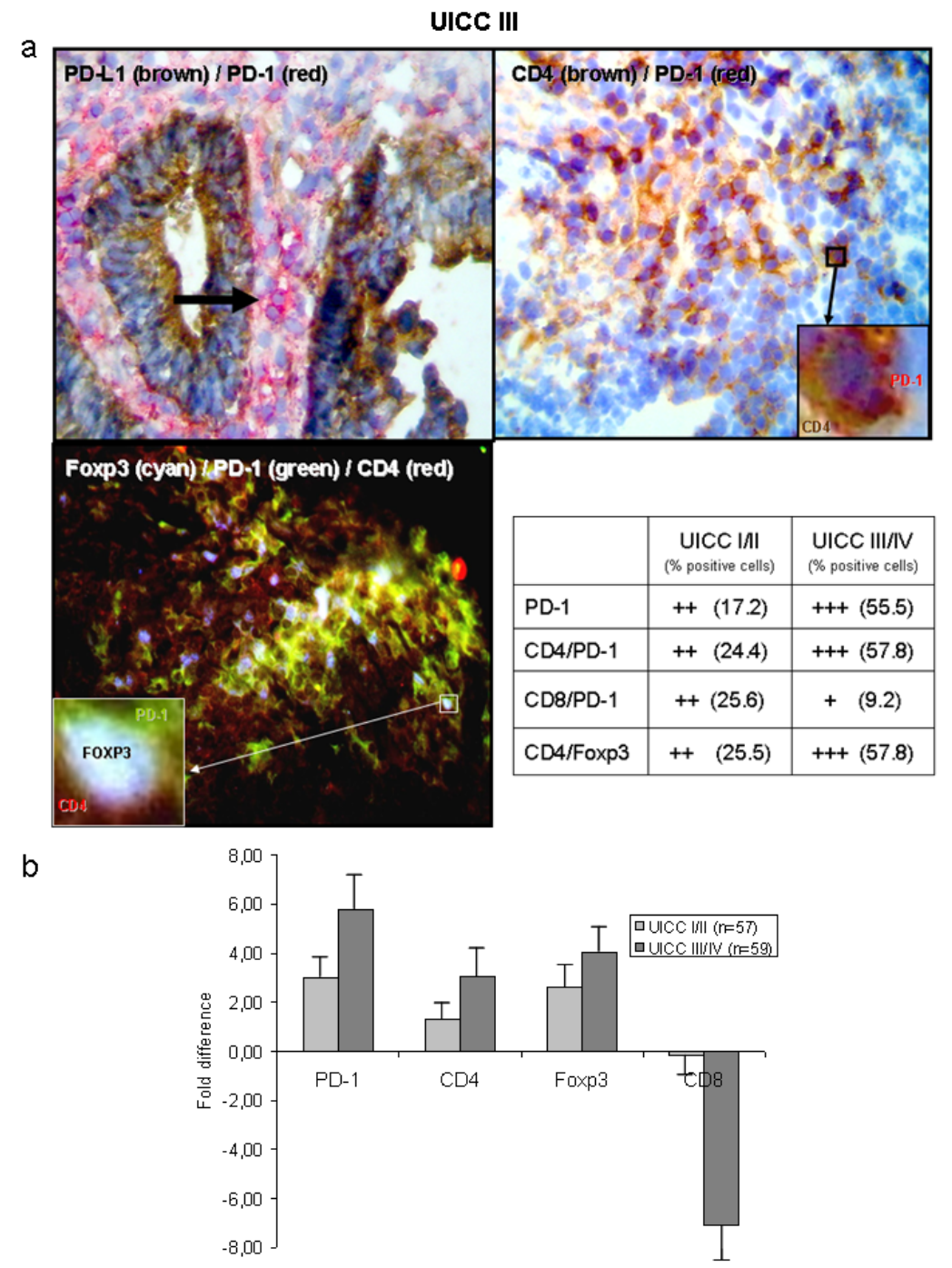

Figure 3: Immunohistochemical, immunofluorescence staining, and quantification of PD-L gene expression by real-time PCR of colorectal tissues. Representative example of PD-1/PD-L2, PD-1/CD4, and PD-1/CD4/Foxp3 infiltrating T lymphocytes (a). Expression of PD-1 (red, arrow) was primarily detectable on infiltrating T lymphocytes, while PD-L1 (brown) was mainly expressed on tumor cells of patients at UICC stage III (top left). PD-1 was mainly present on CD4 positive T cells (top right and bottom right). Immunofluorescence analysis showed strong PD-1 expression on CD4/Foxp3 infiltrating T lymphocytes at UICC stage III (bottom left). Expression of PD-1 on CD8+ T cells was mainly observed at UICC stages I/II (bottom right). Increased expression of CD4/Foxp-3 positive regulatory T cells was detected of PD-1 on CD8+ T cells was mainly observed at UICC stages I/ll (bottom right). Increased expression of CD4/Foxp-3 positive regulatory T cells was detected
during cancer progression (bottom right) (a). Immunohistochemical staining: $3.3^{\prime}$-diaminobenzidine (DAB), brown colour; Fast Red (red colour). Immunofluorescence staining: Cy5, cyan; FITC (Fluorescein-5-isothiocyanate), green; PE, (Phycoerythrin), red; 400x detailed micrograph. PD-1, CD4, and Foxp-3 mRNA expression was significantly increased at the tumor site in patients with colorectal cancer. CD8 gene expression was decreased at the tumor site of those patients (b).

positive immune cells become negatively controlled through PDL1 and/or PD-L2 signaling cancer cells within a specific tumor microenvironment and possibly by additional $\mathrm{T}$ cells of regulatory behavior.

\section{Discussion}

The inability of $\mathrm{T}$ cells to recognize tumor cells due to an impaired antigen presentation on the tumor cell surface as well as an inhibition of $\mathrm{T}$ cell activation by immunosuppressive proteins at the tumor site are mechanisms which preclude an effective anti-tumor immune response. This permits further tumor growth and metastasis. Tumorinfiltrating $\mathrm{T}$ lymphocytes are considered as a manifestation of the host immune response [27]. Particularly CD8-positive effector T cells with help of specific CD4-positive helper T cells infiltrating the tumor are contributing to the elimination of tumor cells. However, infiltrating $\mathrm{T}$ lymphocytes could also have negative regulatory functions particularly on effector immune cells, thus, contributing to tumor growth and metastasis. Several clinical studies have suggested that tumorinfiltrating $\mathrm{T}$ lymphocytes play a critical role and may have prognostic significance in certain human tumors including colorectal cancer [28].

The negative regulatory programmed death-1/programmed death ligand (PD-1/PD-L) pathway in $\mathrm{T}$ cell activation has been suggested to play an important role in tumor evasion from host immunity by engaging PD-1 receptors on activated T and B cells [14,29]. In this study both ligands, PD-L1 and PD-L2, were found to be expressed in CRC. Interestingly, patients with PD-L negative tumors showed a significantly better survival than those expressing either PD-L1 or 
PD-L2. Moreover, the impact of overexpressing one of the ligands (either PD-L1 or PD-L2) as determined on the gene level was different between patients at advanced cancer stages with nodal involvement (N1/2) and distant metastasis (M1). Multivariate analysis showed that $\mathrm{PD}-\mathrm{L} 1 / \mathrm{PD}-\mathrm{L} 2 \mathrm{mRNA}$ overexpression was an independent prognostic factor negatively influencing the prognosis of patients with CRC. Thus, our results demonstrate PD-L gene overexpression as an independent prognostic factor in CRC and confirm clinical data in solid tumors [30-33]. Consequently, PD-L expression is suggestive to be involved in downregulating anti-tumor immunity and thus promoting further tumor growth and metastasis in patients with CRC.

The herein presented results from clinical CRCs support the hypothesis that expression of PD-L1, as recently supposed to be predominantly tumor cell-mediated, promotes cancer progression through downregulation of tumor-reactive $\mathrm{T}$ cells [18]. Previous experimental findings strengthen this theory. However, PD-L1 expression in CRC has not been fully addressed so far. Nevertheless, a strong correlation between PD-L1 expression on tumor cells and discrepant clinical outcomes has been observed [30,33]. Thus, the predictive value of $\mathrm{PD}-\mathrm{L} 1$ expression on tumor cells is still controversial in CRC patients. However, we cannot ignore the value of PD-L1 expression in selecting patients who will benefit from immunotherapy. Some clinical trials have demonstrated that immunotherapy significantly improves progression-free survival in patients.

We have shown that patients with invasion of $\mathrm{PD}-1$ positive immune cells in their colorectal cancers developed significantly more often concomitant PD-L expression in their tumors. In fact, the expression of PD-1 on infiltrating immune cells in tumors of patients with CRC was significantly upregulated at late stages of the disease. Regulatory $\mathrm{T}$ cells are capable of inhibiting the activity of other cells, including CD4- and CD8-positive T cells [23,34-36]. Interestingly, the number of infiltrating $\mathrm{T}$ cells with regulatory characteristics (CD4- and Foxp3 positivity) significantly increased in tumor tissues of our analyzed cancer patients from early to advanced tumor stages. This data are in accordance with the observation that a blockade of PD-L1 led to a decreased number of regulatory $\mathrm{T}$ cells [37]. Regulatory $\mathrm{T}$ cells can also suppress the function of cytotoxic CD8-positive T cells, thus inhibiting anti-tumor immune responses, largely through a mechanism that requires cell-cell contact. CD8-positive $\mathrm{T}$ cells are generally thought to play a central role in anti-tumor immune responses and the presence of CD8-positive $\mathrm{T}$ cells has been reported as a prognostic factor [38-44]. In our study, we observed an inverse correlation between PD-L overexpression in the tumors and CD8-positive T lymphocyte infiltration mainly in patients with late stage cancers indicating that PD-L expression can negatively influence the presence of CD8-positive T cells. Interestingly we found further evidence from our analyzed colorectal cancers for co-expression of PD-1 on CD4/Foxp3-positive T cells. This indicates $\mathrm{T}$ regulatory cell-mediated mechanisms by which tumor cells can evade immune recognition and destruction $[19,23]$. These results are in accordance with the recent work by Masugi et al. The authors had observed a significant inverse association of tumor PD-L1 expression with Foxp3+ cell density, but not with CD8+ T cell density, suggesting that the influence of tumor-mediated PD-L1 expression on T-cell densities vary in the different $\mathrm{T}$ cell subpopulations [22]. Altogether, this indicates interactive roles of the immune checkpoint pathway and host immunity in CRC.

Multivariate analysis of patient and tumor characteristics including PD-L1/PD-L2 overexpression in colorectal cancers in our patient cohort with a completed 5-year follow-up strongly suggests that PD-1 is of prognostic relevance for patients with CRC.

\section{Conclusion}

The results of the current study suggest that PD-1/PD-L1/PD-L2 tumor-immune cell signaling events are promoting cancer progression in patients with CRC. PD-1/PD-L mediated inhibitory signaling gives a selective growth advantage for the cancer by inhibiting CD8-positive cytotoxic $\mathrm{T}$ cell responses and augmenting the function of regulatory $\mathrm{T}$ cells. Our data including the tumor immunity status and relevant clinicopathological and tumor molecular results elucidate potential interactive roles of the immune checkpoint pathway and the host immunity. It will be necessary to design prospective trials to validate predictive factors to select patients with colorectal cancer with the highest chance to benefit from immune agents.

\section{Conflict of Interest}

All authors confirm that they have no conflicts of interest concerning this study.

\section{Acknowledgments}

This work was supported by the Deutsche Bundesstiftung Umwelt grant (no DBU 16011) for Scientific Research, Germany. The authors thank the assistance of Mariola Dragan, Sybille Eber, Sabine Mueller-Morath, Ingrid Strauss, and Andrea Trumpfheller for their technical support.

\section{References}

1. Wiggers T, Arends JW, Schutte B, Volovics L, Bosman FT (1988) A multivariate analysis of pathologic prognostic indicators in large bowel cancer. Cancer 61: 386-395.

2. Hollstein M, Sidransky D, Vogelstein B, Harris CC (1991) p53 mutations in human cancers. Science 253: 49-53.

3. Medical Research Council Oesophageal Cancer Working Group (2002) Surgical resection with or without preoperative chemotherapy in oesophageal cancer: A randomised controlled trial. Lancet 359: 1727-1733.

4. Kelsen DP, Ginsberg R, Pajak TF, Sheahan DG, Gunderson L, et al. (1998) Chemotherapy followed by surgery compared with surgery alone for localized esophageal cancer. N Engl J Med 339: 1979-1984.

5. Arnott SJ, Duncan W, Gignoux M, Girling DJ, Hansen HS, et al. (1998) Preoperative radiotherapy in esophageal carcinoma: A meta-analysis using individual patient data (Oesophageal Cancer Collaborative Group). Int J Radiat Oncol Biol Phys 41: 579-583.

6. Hanson HL, Donermeyer DL, Ikeda H, White JM, Shankaran V, et al. (2000) Eradication of established tumors by CD8+ T cell adoptive immunotherapy. Immunity 13: 265-276.

7. Lee PP, Yee C, Savage PA, Fong L, Brockstedt D, et al. (1999) Characterization of circulating $T$ cells specific for tumor-associated antigens in melanoma patients. Nat Med 5: 677-685.

8. Molldrem JJ, Lee PP, Wang C, Felio K, Kantarjian HM, et al. (2000) Evidence that specific $T$ lymphocytes may participate in the elimination of chronic myelogenous leukemia. Nat Med 6: 1018-1023.

9. Romero P, Dunbar PR, Valmori D, Pittet M, Ogg GS, et al. (1998) Ex vivo staining of metastatic lymph nodes by class I major histocompatibility complex tetramers reveals high numbers of antigen-experienced tumor-specific cytolytic T lymphocytes. J Exp Med 188: 1641-1650.

10. Ishida Y, Agata Y, Shibahara K, Honjo T (1992) Induced expression of PD-1, novel member of the immunoglobulin gene superfamily, upon programmed cell death. Embo J 11: 3887-3895.

11. Nishimura H, Honjo T (2001) PD-1: An inhibitory immunoreceptor involved in peripheral tolerance. Trends Immunol 22: 265-268.

12. Okazaki T, Chikuma S, Iwai Y, Fagarasan S, Honjo T (2013) A rheostat for immune responses: The unique properties of PD-1 and their advantages for clinical application. Nat Immunol 14: 1212-1218.

13. Dong H, Zhu G, Tamada K, Chen L (1999) B7-H1, a third member of the B7 family, co-stimulates T-cell proliferation and interleukin-10 secretion. Nat Med 5: $1365-1369$. 
14. Freeman GJ, Long AJ, Iwai Y, Bourque K, Chernova T, et al. (2000) Engagement of the PD-1 immunoinhibitory receptor by a novel B7 family member leads to negative regulation of lymphocyte activation. J Exp Med 192: 1027-1034.

15. Tseng SY, Otsuji M, Gorski K, Huang X, Slansky JE, et al. (2001) B7-DC, a new dendritic cell molecule with potent costimulatory properties for $T$ cells. $J$ Exp Med 193: 839-846.

16. Keir ME, Butte MJ, Freeman GJ, Sharpe AH (2011) PD-1 and its ligands in tolerance and immunity. Annu Rev Immunol 26: 677-704.

17. Latchman Y, Wood CR, Chernova T, Chaudhary D, Borde M, et al. (2001) PD-L2 is a second ligand for PD-1 and inhibits T cell activation. Nat Immunol 2: 261-268.

18. Dong H, Strome SE, Salomao DR, Tamura H, Hirano F, et al. (2002) Tumorassociated $\mathrm{B} 7-\mathrm{H} 1$ promotes $\mathrm{T}$-cell apoptosis: A potential mechanism of immune evasion. Nat Med 8: 793-800.

19. Krupnick AS, Gelman AE, Barchet W, Richardson S, Kreisel FH, et al. (2005) Murine vascular endothelium activates and induces the generation of allogeneic CD4+25+Foxp3+ regulatory T cells. J Immunol 175: 6265-6270.

20. Iwai $Y$, Ishida M, Tanaka Y, Okazaki T, Honjo T, et al. (2002) Involvement of PD-L1 on tumor cells in the escape from host immune system and tumor immunotherapy by PD-L1 blockade. Proc Natl Acad Sci USA 99: 12293-12297.

21. Motzer RJ, Escudier B, McDermott DF, George S, Hammers HJ, et al. (2015) Nivolumab versus Everolimus in advanced renal-cell carcinoma. N Engl J Med 373: 1803-1813.

22. Masugi Y, Nishihara R, Yang J, Mima K, Da Silva A, et al. (2017) Tumour CD274 (PD-L1) expression and T cells in colorectal cancer. Gut 66: 1463-1473.

23. Kim M, Grimmig T, Grimm M, Lazariotou M, Meier E, et al. (2013) Expression of Foxp3 in colorectal cancer but not in Treg cells correlates with disease progression in patients with colorectal cancer. PLoS One 8: e53630.

24. Wang X, Teng F, Kong L, Yu J (2016) PD-L1 expression in human cancers and its association with clinical outcomes. Onco Targets Ther 9: 5023-5039.

25. Liu X, Gao JX, Wen J, Yin L, Li O, et al. (2003) B7DC/PDL2 promotes tumor immunity by a PD-1-independent mechanism. J Exp Med 197: 1721-1730.

26. Radhakrishnan S, Nguyen LT, Ciric B, Flies D, Van Keulen VP, et al. (2004) Immunotherapeutic potential of B7-DC (PD-L2) cross-linking antibody in conferring antitumor immunity. Cancer Res 64: 4965-4972.

27. Rosenberg SA (1996) The immunotherapy of solid cancers based on cloning the genes encoding tumor-rejection antigens. Annu Rev Med 47: 481-491.

28. Koch M, Beckhove P, Op Den Winkel J, Autenrieth D, Wagner P, et al. (2006) Tumor infiltrating $T$ lymphocytes in colorectal cancer: Tumor-selective activation and cytotoxic activity in situ. Ann Surg 244: 986-992.

29. Okazaki T, Maeda A, Nishimura H, Kurosaki T, Honjo T (2001) PD-1 immunoreceptor inhibits B cell receptor-mediated signaling by recruiting src homology 2-domain-containing tyrosine phosphatase 2 to phosphotyrosine. Proc Natl Acad Sci USA 98: 13866-13871.
30. Shi SJ, Wang LJ, Wang GD, Guo ZY, Wei M, et al. (2013) B7-H1 expression is associated with poor prognosis in colorectal carcinoma and regulates the proliferation and invasion of HCT116 colorectal cancer cells. PLoS One 8: e76012.

31. Francisco LM, Sage PT, Sharpe AH (2010) The PD-1 pathway in tolerance and autoimmunity. Immunol Rev 236: 219-242.

32. Wu C, Zhu Y, Jiang J, Zhao J, Zhang XG, et al. (2006) Immunohistochemical localization of programmed death-1 ligand-1 (PD-L1) in gastric carcinoma and its clinical significance. Acta Histochem 108: 19-24.

33. Droeser RA, Hirt C, Viehl CT, Frey DM, Nebiker C, et al. (2013) Clinical impact of programmed cell death ligand 1 expression in colorectal cancer. Eur $J$ Cancer 49: 2233-2242.

34. Ramsdell F (2003) Foxp3 and natural regulatory T cells: Key to a cell lineage? Immunity 19: 165-168.

35. Sakaguchi $S$ (2004) Naturally arising CD4+ regulatory $t$ cells for immunologic self-tolerance and negative control of immune responses. Annu Rev Immunol 22: 531-562.

36. Shevach EM (2002) CD4+ CD25+ suppressor T cells: More questions than answers. Nat Rev Immunol 2: 389-400.

37. Tanaka K, Albin MJ, Yuan X, Yamaura K, Habicht A, et al. (2007) PDL1 is required for peripheral transplantation tolerance and protection from chronic allograft rejection. J Immunol 179: 5204-5210.

38. Cho Y, Miyamoto M, Kato K, Fukunaga A, Shichinohe T, et al. (2003) CD4+ and $C D 8+T$ cells cooperate to improve prognosis of patients with esophageal squamous cell carcinoma. Cancer Res 63: 1555-1559.

39. Schumacher K, Haensch W, Roefzaad C, Schlag PM (2001) Prognostic significance of activated $\mathrm{CD} 8(+) \mathrm{T}$ cell infiltrations within esophageal carcinomas. Cancer Res 61: 3932-3936.

40. Nosho K, Baba Y, Tanaka N, Shima K, Hayashi M, et al. (2010) Tumourinfiltrating T-cell subsets, molecular changes in colorectal cancer, and prognosis: Cohort study and literature review. J Pathol 222: 350-366.

41. Galon J, Costes A, Sanchez-Cabo F, Kirilovsky A, Mlecnik B, et al. (2006) Type, density, and location of immune cells within human colorectal tumors predict clinical outcome. Science 313: 1960-1964.

42. Mlecnik B, Bindea G, Angell HK, Sasso MS, Obenauf AC, et al. (2014) Functional network pipeline reveals genetic determinants associated with in situ lymphocyte proliferation and survival of cancer patients. Sci Transl Med 6: 228 ra37.

43. Shang B, Liu Y, Jiang SJ, Liu Y (2015) Prognostic value of tumor-infiltrating FoxP3+ regulatory T cells in cancers: A systematic review and meta-analysis. Sci Rep 5: 15179

44. Mei Z, Liu Y, Liu C, Cui A, Liang Z, et al. (2014) Tumour-infiltrating inflammation and prognosis in colorectal cancer: Systematic review and meta-analysis. $\mathrm{Br}$ Cancer 110: 1595-1605. 